\title{
Clinical pattern of inflammatory bowel disease in Southern Saudi Arabia: a retrospective cohort study
}

Mohammed Attieh Alzahrani (1)

Mushabab Ali M. Alshahrani (2)

Ayman Ali Ahmed Alshahrani (2)

Abduallah Mohammed Abdullah Shahrani (2)

Hanady Mohammed Awad Alhumaidi (2)

Huda Saleh Alsharbi (2)

Mushabab Saeed Hammad (3)

Khalid Yousef Nebrawi (3)

Abdullah Mohammed Alshehri (3)

Shehata F. Shehata $(4,5)$

Suliman Mohammed Alhumayed (6)

Mohammed Elbagir K Ahmed (6)

(1) Assistant professor, Department of Medicine, College of Medicine, King Khalid University, Abha, Saudi Arabia

(2) Medical Resident, Department of Medicine, Aseer Central Hospital, Abha, Saudi Arabia

(3) Gastroenterology Consultant, Gastroenterology Division, Aseer Central Hospital, Abha, Saudi Arabia

(4) Assistant Professor, College of Medicine, King Khalid University, Abha, Saudi Arabia

(5) Lecturer of Biostatistics, High Institute of Public Health Alexandria University, Egypt

(6) Professor of Medicine, Department of Medicine, college of medicine, King Khalid University, Abha, Saudi Arabia

Corresponding author:

Mohammed Attieh Alzahrani

Assistant Professor

Internal Medicine Department,

King Khalid University

Abha- Saudi Arabia

P.O. BOX 641

Phone : 00966504586134

Email: mohammedalzahrani641@gmail.com;dr_mohdaz@hotmail.com

Received September 2020. Accepted October 2020. Published November 1, 2020.

Please cite this article as: Mohammed Attieh Alzahrani et al. Clinical pattern of inflammatory bowel disease in Southern Saudi Arabia: a retrospective cohort study. Middle East J Intern Med 2020; 13(2): 13-19. DOI: 10.5742/MEJIM2020.93784 


\section{ABSTRACT}

Background \& Study Aim: Inflammatory bowel disease (IBD) is characterized by chronic inflammation of the gastrointestinal tract, caused by a dysregulated immune response to the host intestinal microflora. The main types of IBD are ulcerative colitis (UC) and Crohn's disease (CD). Ulcerative colitis causes long-lasting inflammation and ulceration in the innermost linings of the large intestine (colon) and rectum, while Crohn's disease is characterized by inflammation of the lining of the digestive tract. The present study was conducted to assess magnitude, epidemiological pattern, and complications of IBD in Aseer region of Southern Saudi Arabia.

Patients \& Methods: A retrospective study targeting patient records of all patients with IBD, who were diagnosed with $C D$ and $U C$ over the period between 1980 and 2019 at gastroenterology unit, Aseer central hospital. The data were extracted from the patients' files covering the demographic data, signs and symptoms at the onset of disease, laboratory data, radiological findings, and endoscopic findings.
Results: The study included 134 patients, of whom $68(50.7 \%)$ were diagnosed with ulcerative colitis (UC) and $66(49.3 \%)$ with Crohn's disease (CD). Considering the endoscopic findings, the $C D$ patients have maximum site of lesion at terminal ileum, followed by the descending and sigmoid colon. Whereas in UC patients the most recorded endoscopic site affected was the descending colon followed by sigmoid colon, and then transverse colon. Anemia was the most frequent complication affecting both groups of patients.

Conclusion: IBD is no longer an uncommon ailment in KSA. UC is in a sound state, while CD is escalating considerably and far outnumbering $U C$.

Key words: Inflammatory bowel diseases (IBD), ulcerative colitis (UC), Crohn's disease (CD), Computed Tomography (CT), Magnetic Resonance Imaging (MRI)

\section{Introduction}

Inflammatory bowel disease (IBD) is a chronic inflammatory disorder affecting gastrointestinal (GI) tract, mainly small and large intestine. There are two major types of IBD: Crohn's disease (CD) and ulcerative colitis (UC). However, there is a small group $(10 \%)$ of patients who are not classified as having either CD or UC; those are categorized as intermediate colitis $(1,2)$. The exact cause of IBD is unknown but there are many current hypotheses implicating a combination of genetic, environmental and immunoregulatory factors in pathogenesis of IBD (1, $3)$. The infectious microbes, ethnic origin, genetic susceptibility, and a dysregulated immune system can result in mucosal inflammation. The incidence and prevalence of IBD are higher in the northern part of the world such as Europe and Canada. However, recent studies have reported an increase in the incidence and prevalence of IBD in the Middle East and Gulf countries which were previously known to have low incidence and prevalence $(4,5,6)$. A few reports from Saudi Arabia, mainly from Riyadh, have shown an increase of the incidence and prevalence of $\operatorname{IBD}(7,8,9)$. This increase has been attributed to the westernization of the lifestyle and improvement in sanitization (10).

Both CD and UC are commonly diagnosed before the age of 30 years, although a small group is detected at an older age between $50 \mathrm{~s}$ and $60 \mathrm{~s}(11)$. CD usually affects any part of the gastro-intestinal tract from mouth to anus. Smoking has been reported to reduce the risk of $\mathrm{UC}$, but in contrast, increases the risk for CD. Furthermore, previous reports documented a slight gender difference as $\mathrm{CD}$ has been reported to be higher in women, whereas UC is reported to be higher in the male population $(9,12)$.
$\mathrm{CD}$ and $\mathrm{UC}$ are chronic, relapsing diseases in which symptoms vary from patient to patient and may range from mild to severe in intensity. The commonly experienced symptoms are dysentery, abdominal pain, weight loss, and arthralgia $(9,10$, 11). Once IBD has been diagnosed, the symptoms can often be effectively managed. However, CD and UC are chronic illness where disease may recur and complications may flare up.

The aim of this study was to estimate the prevalence of IBD in Aseer region and to study the demographic, clinical pattern, and course of the disease in terms of therapeutic management with medications and surgical interventions.

\section{Patients and Methods}

This is a retrospective record based study targeting all records of 134 patients with IBD, who were diagnosed with either CD or UC over a period from 1980 to 2019 at the gastroenterology unit, Asser central hospital (ACH), Abha, Saudi Arabia. This is the main tertiary hospital in Aseer region, southern part of Saudi Arabia, serving more than two million people. The hospital has a total clinical capacity of 574 beds, and contains the latest modern medical devices and technologies that rarely present in most of the hospitals in some of the developed countries. The data were extracted with the help of specially designed data collection tools to minimize extraction errors. The data extracted covered the demographic details of the patients, signs and symptoms at the onset of the disease, date of onset, time interval between the disease onset and the time of diagnosis, laboratory, and radiological and endoscopic findings. The present study was approved by the Ethics Committee of Aseer Central hospital. 


\section{Statistical analysis}

The data were extracted, revised, coded and fed into statistical software IBM SPSS version 22. All statistical analysis was performed using two tailed tests and alpha error of 0.05 and $\mathrm{P}$ value less than or equal to 0.05 was considered to be statistically significant. Descriptive analysis is based on frequency and percent distribution for all patients' demographics, clinical data, radiological and endoscopic findings.

\section{Results}

\section{Epidemiology of patients with IBD}

The study included 134 patients of whom 68 (50.7\%) were diagnosed with ulcerative colitis (UC) and 66 (49.3\%) with Crohn's disease (CD). For patients with Crohn's disease, $65.2 \%$ were below the age of 30 years and $66.7 \%$ were men. A total $15.2 \%$ overweight patients were recorded and $89.4 \%$ of them had the disease for 9 years or less. Regarding ulcerative colitis, $45.6 \%$ were aged less than 30 years, and $54.4 \%$ were men. Obesity was recorded among $11.8 \%$ of the cases and $86.8 \%$ had the disease for 9 years or less (Table 1).

\section{Clinical presentation of patients with IBD}

Table (2) demonstrates the clinical presentation of the study population. The most recorded physical signs among CD cases were abdominal tenderness (36.4\%) followed by skin lesion $(21.2 \%)$, and pallor $(18.2 \%)$. On the other hand, the frequency of the same physical signs among patients with UC was $33.8 \%, 29.4 \%$, and $25 \%$ respectively.

Considering symptoms, the most reported symptoms by patients with CD were abdominal pain (87.9\%), followed by weight loss (47\%), and bloody diarrhea (42.4\%), while the frequency of these symptoms reported by UC patients were: $75 \%, 64.7 \%$, and $33.8 \%$ respectively.

\section{Radiological findings}

According to radiological findings, the most frequent affected sites detected by CT in CD cases were: ileum (67.4\%), sigmoid colon (37.2\%), ileocecal $(30.2 \%)$, and jejunum $(25.6 \%)$. In contrast, in UC, the lesions were seen in sigmoid colon $(72.2 \%)$, descending colon $(66.7 \%)$, transverse colon (55.6\%), and ascending colon (27.8\%). Specific CT pathological findings reported among $\mathrm{CD}$ cases was wall thickening which was found in the majority of the cases $(84.6 \%)$. This was followed by wall enhancement (15.4\%), and abscess formation (12.8\%). However, in UC, the reported corresponding CT findings were $80 \%$, $20 \%$, and $13.3 \%$ respectively. MRI was done in only 25 patients who showed that the most affected site of lesion among patients with CD were: rectum (40\%), anus (26.7\%), and then sigmoid colon $(26.7 \%)$. While in UC, all lesions detected with MRI were confined to the rectum. Details of MRI pathological findings in CD patients included wall thickening (62.5\%), wall enhancement (37.5\%), and perianal fistula $(25 \%)$. However, the frequencies of these abnormal MRI findings in UC patients were evenly distributed at $50 \%$.

\section{Disease behavior and extent of the disease}

The endoscopic findings are shown in Table 3. It is clear that terminal ileum was the main site affected (41.2\%) among CD cases. Other affected sites were the descending, sigmoid colon (35.3\% for each), and the ileocecal valve (31.4\%). It was alarming that the majority of patients with UC $(69.65 \%)$ had pan-colitis, while $21.4 \%$ had proctitis, and $8.9 \%$ had left sided colitis.

\section{Medical management}

As shown in Table 4, the majority of patients with CD (70\%) received Azathioprine in addition to biological therapy and calcium supplement in $50 \%$ of them, while $43.3 \%$ received corticosteroids. The corresponding percentages in patients with UC were $59.7 \%$ for both Azathioprine and Amino Salicylates, 64.5\% for calcium supplements, and $50 \%$ for steroids.

\section{Complication of the disease}

The spectrum of complications (Table 5) was as follows: anemia (58.1\%) among patients with CD and $79.2 \%$ of UC patients. Moreover, $35.5 \%$ of UC patients had toxic mega colon and $32.3 \%$ of CD patients had intestinal obstruction. 
Table 1: Personal characteristics of patients with inflammatory bowel diseases in Aseer Central Hospital

\begin{tabular}{lcccc}
\hline & \multicolumn{4}{c}{ Diagnosis } \\
\cline { 2 - 5 } Personal data & \multicolumn{2}{c}{$\begin{array}{c}\text { Crohn's Disease } \\
(\mathrm{n}=66)\end{array}$} & $\begin{array}{c}\text { Ulcerative colitis } \\
(\mathrm{n}=68)\end{array}$ \\
\cline { 2 - 6 } & No & $\%$ & No & $\%$ \\
\hline Age (in years) & & & & \\
$\quad<30$ years & 43 & $65.2 \%$ & 31 & $45.6 \%$ \\
$30-39$ & 14 & $21.2 \%$ & 17 & $25.0 \%$ \\
$\quad 40+$ & 9 & $13.6 \%$ & 20 & $29.4 \%$ \\
\hline Sex & & & & \\
$\quad$ Male & 44 & $66.7 \%$ & 37 & $54.4 \%$ \\
$\quad$ Female & 22 & $33.3 \%$ & 31 & $45.6 \%$ \\
\hline Body mass index & & & & \\
$\quad$ Normal & 56 & $84.8 \%$ & 60 & $88.2 \%$ \\
$\quad$ Overweight/ obese & 10 & $15.2 \%$ & 8 & $11.8 \%$ \\
\hline Disease duration & & & & \\
(in years) & & & & \\
$1-4$ & 20 & $30.3 \%$ & 14 & $20.6 \%$ \\
$\quad 5-9$ & 39 & $59.1 \%$ & 45 & $66.2 \%$ \\
$\quad 10+$ & 7 & $10.6 \%$ & 9 & $13.2 \%$ \\
\hline
\end{tabular}

Table 2: Clinical presentation of cases of IBD in Aseer Central Hospital

\begin{tabular}{llcccc}
\hline \multirow{3}{*}{ Clinical presentation } & & \multicolumn{3}{c}{ Diagnosis } \\
\cline { 3 - 6 } & & Crohn's Disease & \multicolumn{2}{c}{ Ulcerative colitis } \\
\cline { 3 - 6 } & & No & $\%$ & No & $\%$ \\
\hline \multirow{5}{*}{ Physical sign at } & Aphous ulcer & 1 & $1.5 \%$ & 1 & $1.5 \%$ \\
& Abdominal tenderness & 24 & $36.4 \%$ & 23 & $33.8 \%$ \\
& Abdominal mass & 6 & $9.1 \%$ & 6 & $8.8 \%$ \\
& Eye signs & 8 & $12.1 \%$ & 4 & $5.9 \%$ \\
& Skin lesion & 14 & $21.2 \%$ & 20 & $29.4 \%$ \\
& Pallor & 12 & $18.2 \%$ & 17 & $25.0 \%$ \\
& Other & 10 & $15.2 \%$ & 2 & $2.9 \%$ \\
\hline \multirow{5}{*}{ Symptom } & 58 & $87.9 \%$ & 51 & $75.0 \%$ \\
& Abdominal pain & 28 & $42.4 \%$ & 44 & $64.7 \%$ \\
& Bloody diarrhea & 7 & $10.6 \%$ & 11 & $16.2 \%$ \\
& Rectal bleeding & 31 & $47.0 \%$ & 23 & $33.8 \%$ \\
& Weight Ioss & 12 & $18.2 \%$ & 1 & $1.5 \%$ \\
& Constipation & 3 & $4.5 \%$ & 6 & $8.8 \%$ \\
& Tenesmus & 13 & $19.7 \%$ & 1 & $1.5 \%$ \\
& Fever & 3 & $4.5 \%$ & 5 & $7.4 \%$ \\
& Joint pain & 5 & $7.6 \%$ & 0 & $0.0 \%$ \\
& Mouth ulcer & 13 & $19.7 \%$ & 4 & $5.9 \%$ \\
& Nausea/vomiting & 3 & $4.5 \%$ & 4 & $5.9 \%$ \\
\hline
\end{tabular}


Table 3: Endoscopic clinical findings for cases of IBD in Aseer Central Hospital

\begin{tabular}{lcccc}
\hline \multirow{2}{*}{ Endoscopy findings } & \multicolumn{2}{c}{ Crohn's Disease } & \multicolumn{2}{c}{ Ulcerative colitis } \\
\cline { 2 - 5 } & No & $\%$ & No & $\%$ \\
\hline Terminal Ileum & 21 & $41.2 \%$ & 4 & $7.1 \%$ \\
Ileocecal valve & 16 & $31.4 \%$ & 4 & $7.1 \%$ \\
Cecum & 10 & $19.6 \%$ & 12 & $21.4 \%$ \\
Ascending colon & 13 & $25.5 \%$ & 25 & $44.6 \%$ \\
Transverse colon & 13 & $25.5 \%$ & 38 & $67.9 \%$ \\
Descending colon & 18 & $35.3 \%$ & 41 & $73.2 \%$ \\
Sigmoid & 18 & $35.3 \%$ & 40 & $71.4 \%$ \\
Rectum & 14 & $27.5 \%$ & 33 & $58.9 \%$ \\
Anus & 13 & $25.5 \%$ & 9 & $16.1 \%$ \\
\hline
\end{tabular}

Table 4: Treatment received by cases of IBD in Aseer Central Hospital

\begin{tabular}{lcccc}
\hline \multirow{2}{*}{ Treatment received } & \multicolumn{4}{c}{ Diagnosis } \\
\cline { 2 - 5 } & \multicolumn{2}{c}{ Crohn's Disease } & \multicolumn{2}{c}{ Ulcerative colitis } \\
\cline { 2 - 5 } & No & $\%$ & No & $\%$ \\
\hline Steroid & 26 & $43.3 \%$ & 31 & $50.0 \%$ \\
Azathioprine (Imuran) & 42 & $70.0 \%$ & 37 & $59.7 \%$ \\
Aminosalcylates (5-ASA) & 22 & $36.7 \%$ & 37 & $59.7 \%$ \\
Metronidazole & 1 & $1.7 \%$ & 2 & $3.2 \%$ \\
Ciprofloxacin & 3 & $5.0 \%$ & 1 & $1.6 \%$ \\
Methotrexate (MTX) & 1 & $1.7 \%$ & 2 & $3.2 \%$ \\
Biological therapy & 30 & $50.0 \%$ & 23 & $37.1 \%$ \\
Iron supplements & 9 & $15.0 \%$ & 12 & $19.4 \%$ \\
Calcium supplement & 30 & $50.0 \%$ & 40 & $64.5 \%$ \\
\hline
\end{tabular}

Table 5: Complications recorded among cases of IBD in Aseer Central Hospital

\begin{tabular}{lcccc}
\hline \multirow{2}{*}{ Complications } & \multicolumn{4}{c}{ Diagnosis } \\
\cline { 2 - 5 } & \multicolumn{3}{c}{ Crohn's Disease } & Ulcerative colitis \\
\cline { 2 - 5 } & No & $\%$ & No & $\%$ \\
\hline Anemia & 18 & $58.1 \%$ & 19 & $79.2 \%$ \\
Intestinal obstruction & 10 & $32.3 \%$ & 1 & $4.2 \%$ \\
Sepsis & 4 & $12.9 \%$ & 0 & $0.0 \%$ \\
Toxic mega colon & 11 & $35.5 \%$ & 2 & $8.3 \%$ \\
Stricture & 1 & $3.2 \%$ & 0 & $0.0 \%$ \\
Colon ca & 0 & $0.0 \%$ & 2 & $8.3 \%$ \\
PSC & 0 & $0.0 \%$ & 1 & $4.2 \%$ \\
Fulminant colitis & 2 & $6.5 \%$ & 1 & $4.2 \%$ \\
Bowel perforation & 0 & $0.0 \%$ & 0 & $0.0 \%$ \\
Small bowel & 2 & $6.5 \%$ & 0 & $0.0 \%$ \\
bacterial overgrowth & 0 & $0.0 \%$ & 2 & $8.3 \%$ \\
Osteoporosis & 3 & $9.7 \%$ & 1 & $4.2 \%$ \\
DVT or PE & & &
\end{tabular}




\section{Discussion}

In this study we have tried to present the first hospital-based study that evaluates the prevalence and clinical pattern of IBD in the southern region of Saudi Arabia. IBD is now considered as a global healthcare issue with increasing incidence and prevalence rate in the last 20 years (13). It is our belief that the incidence of IBD is increasing in the Middle East including Saudi Arabia, due to westernization of the lifestyle with regard to eating and living habits $(12,14-16)$. The first reported incidence of IBD in Saudi Arabia was published in 1982 by Mokhtar and Khan estimating the incidence of $\mathrm{CD}$ as very low at $0.32 / 100,000$ population(17). This low incidence was supported by El-Mouzan et al. in 2014 who reported that the prevalence of CD in Saudi Arabia in children younger than 14 to be only $0.27 / 100,000$ population (18). Furthermore, a recent review by Taghreed reported that the incidence and prevalence of IBD in Saudi Arabia is increasing but an accurate estimate is lacking due to the limited studies in this condition (19). In our study, the ratio of patients diagnosed with UC to those diagnosed with CD was approximately $1: 1$, which is in contrast to the results observed from other countries, where UC was much more common than CD (20-24). This new observation has been supported by earlier studies which indicated that $\mathrm{CD}$ incidence is on the rise in Saudi Arabia due to the great changes in eating habits and a life style mimicking that of western countries $(25,26)$.

It is worth mentioning, that we found a male dominance in both patients with UC and patients with $\mathrm{CD}$, in contrast to the equal sex distribution reported from other areas of Saudi Arabia and from some Asian countries(20, 21).On the other hand, IBD may occur at any age, mostly between 15-30 years; the majority of our patients with $\mathrm{CD}$ were younger than 30 years, in agreement with other studies from Saudi Arabia and Asian countries(19, 20, 21)

In our study, the most common clinical presentation reported in patients was abdominal pain followed by weight loss and dysentery which is consistent with what has been reported from other countries $(19,20,24,25)$. However, at the time of presentation, pan colitis was seen in most patients $(69 \%)$ with UC, while, as expected the terminal ileum was the main site affected (41\%) in CD patients. This finding is different from what has been reported from western countries where about $45 \%$ of patients with UC presented with proctosigmoiditis while the small and large bowel involvements were reported in $42 \%$ of patients(26-28).

Considering the therapeutic management, most of the patients with CD received Azathioprine; $43 \%$ patients received steroid at some point of their disease course, and 50\% received antitumor necrosis factor (ANF). However, it is well acknowledged that such issues embrace a personalized approach based not only on disease extent and severity, but also on an individual patient's projected natural history of the disease. It is heartening that about half of our patients received biological therapies based on severity of the disease as recommended by recent guidelines which recommend newer biological therapies such as Tofacitinib, which seems to be most effective in patients who don't respond to infliximab, although vedolizumab may be considered as a reasonable alternative (29-31).
So, finally we can conclude that the ratio of patients diagnosed with UC to those diagnosed with CD was approximately $1: 1$; IBD may occur at any age, mostly between $15-30$ years; the majority of patients with $\mathrm{CD}$ were younger than 30 years.

\section{Study Limitations:}

Certain limitations to this study include the retrospective nature and the difficulty of tracing and follow up of patients to ascertain the rates of remission and mucosal healing.

\section{References}

1. Pierik M, Yang H, Barmada MM, Cavanaugh JA, Annese $\mathrm{V}$, Brant SR. et al. The IBD international genetics consortium provides further evidence for linkage to IBD4 and shows geneenvironment interaction. Inflamm Bowel Dis. 2005; 11:1-7.

2. Wells AD, McMillan I, Price AB, Ritchie JK, Nicholls RJ. Natural history of indeterminate colitis. Br J Surg. 1991; 78:179-81.

3. Loftus EV Jr, Sandborn WJ. Epidemiology of inflammatory bowel disease. Gastroenterol Clin North Am 2002; 31:1-20

4. Cottone M, Cipolla C, Orlando A, Oliva L, Aiala R, Puleo A. Epidemiology of Crohn's disease in Sicily: A hospital incidence study from 1987 to 1989. "The Sicilian Study Group of Inflammatory Bowel Disease”. Eur J Epidemiol 1991; 7:636-40

5. Loftus EV Jr. Clinical epidemiology of inflammatory bowel disease: Incidence, prevalence, and environmental influences. Gastroenterology. 2004; 126:1504-17.

6. Molodecky NA, Soon IS, Rabi DM, Ghali WA, Ferris M, Chernoff $\mathrm{G}$. et al. Increasing Incidence and Prevalence of the Inflammatory Bowel Diseases With Time, Based on Systematic Review. Gastroenterology. 2012; 142:46-54.

7. Al-Ghamdi AS, Al-Mofleh IA, Al-Rashed RS, Al-Amri SM, Al-Jebreen AM, Isnani AC. Epidemiology and outcome of Crohn's disease in a teaching hospital in Riyadh. World J Gastroenterol. 2004; 10:1341-4

8. Al-Mofarreh MA, Al-Mofleh IA, Al-Teimi IN, Al-Jebreen AM. Crohn's disease in Saudi outpatient population: Is it still rare? Saudi J Gastroenterol. 2009; 15:111-6

9. Khawaja AQ, Sawan AS. Inflammatory bowel disease in the Western Saudi Arabia. Saudi Med J 2009; 30:537-40

10. Thia KT, Loftus EV, Jr, Sandborn WJ, Yang SK. An update on the epidemiology of inflammatory bowel disease in Asia. Am J Gastroenterol. 2008; 103:3167-82

11. Cosnes J, Gower-Rousseau C, Seksik P, Cortot A. Epidemiology and natural history of inflammatory bowel diseases. Gastroenterology. 2011; 140(6):1785-1794.

12. Sartir RB. Mechanisms of disease: pathogenesis of Crohn's disease and ulcerative colitis. Nat Clin Pract Gastroenterol Hepatol. 2006; 3(7):390-407.

13. M'Koma AE. Inflammatory bowel disease: An expanding global health problem. Clin Med Insights Gastroenterol 2013; 6:33-47.

14. Ng SC, Bernstein CN, VatnMH, Lakatos PL, Loftus Jr EV, Tysk C, et al. Geographical variability and environmental risk factors in inflammatory bowel disease. Gut 2013; 62:630-49. 
15. Vegh Z, Kurti Z, Lakatos PL. The epidemiology of inflammatory bowel diseases from West to East. J Dig Dis 2017; 18:92-8.

16. Senhaji N, Serrano A, Badre W, et al. Association of inflammatory cytokine gene polymorphisms with inflammatory bowel disease in a Moroccan cohort. Genes Immun 2016; 17:60-5.

17. Mokhtar A and Khan MA. "Crohn's disease in Saudi Arabia”. Saudi Medical Journal 3 (1982): 270-274.

18. El Mouzan MI., et al. "Incidence of pediatric inflammatory bowel disease in Saudi Arabia: A multicenter national study". Inflammatory Bowel Diseases 20.6 (2014): 1085-1090.

19. Taghreed A Hafiz. "Inflammatory Bowel Disease in Saudi Arabia: Challenges and Perspectives". EC Microbiology 15.3 (2019): 217-226.

20. Duphare H, Misra SC, Patnaik PK, Mathur M, Tandon RK. Spectrum of ulcerative colitis in north India. J ClinGastroenterol1994; 18:23-6.

21. Fadda MA., et al. Inflammatory bowel disease in Saudi Arabia: A hospital-based clinical study of 312 patients. Annals of Saudi Medicine.32.3 (2012): 276-282.

22. Aghazadeh R, Zali MR, Bahari A, Amin K, Ghahghaie F, Firouzi F. Inflammatory bowel disease in Iran: A review of 457 cases. J Gastroenterol Hepato 2005; 20:1691-95.

23. Loftus EV, Jr, Sandborn WJ. Epidemiology of inflammatory bowel disease. Gastroenterol Clin North Am 2002; 31:1-20.

24. Al Salamah SM. "Surgery for small bowel Crohn's disease: Experience of a tertiary referral center". Saudi Journal of Gastroenterology 11.2 (2005): 85-92.

25. Al-Mofleh IA and Azzam NA. Crohn's disease Increasing trend in Saudi Arabia; Saudi Medical Journal 34.11 (2013): 1105-1113.

26. Wiercinska-Drapalo A, Jaroszewicz J, Flisiak R, Prokopow- icz D. Epidemiological characteristics of inflammatory bowel disease in North-Eastern Poland. World J Gastroenterol 2005; 11: 2630-2633

27. Langholz E, Munkholm P, Davidsen M, Binder V. Course of ulcerative colitis: Analysis of changes in disease activity over years. Gastroenterology. 1994; 107:3-11.

28. Louis E, Collard A, Oger AF, Degroote E, Aboul Nasr El Yafi FA, Belaiche J. Behaviour of Crohn's disease according to the Vienna classification: Changing pattern over the course of the disease. Gut. 2001; 49:777-82.

29. Regueiro MD, Greer JB, Hanauer SB. Established management paradigms in IBD: treatment targets and therapeutic tools. Am J Gastroenterol Suppl. 2016; 3:8-16.

30. Colombel J-F, Narula N, Peyrin-Biroulet L. Management strategies to improve outcomes of patients with inflammatory bowel diseases. Gastroenterology. 2017; 152(2):351-361.

31. Colombel JF, Panaccione R, Bossuyt P, et al. Effect of tight control management on Crohn's disease (CALM): a multicentre, randomised, controlled phase 3 trial. Lancet. 2018; 390(10114):2779-2789. 